\title{
KALMAN-BASED BLIND MULTIUSER DETECTION WITH MULTIPLE RECEIVER ANTENNAS FOR THE UPLINK OF ASYNCHRONOUS DS-CDMA SYSTEMS
}

\author{
F.P. Wathan, R. Hoshyar, and R. Tafazolli \\ Mobile Communications Research Group \\ CCSR, University of Surrey, United Kingdom \\ $\{$ F Wathan, R. Hoshyar, R. Tafazolli $\} @$ surrey.ac.uk
}

\begin{abstract}
A batch Kalman-based blind adaptive multiuser detection (K-BA-MUD) with multiple receiver (Rx) antennas is investigated for asynchronous CDMA systems in the Uplink direction. In this paper, we consider two receiver structures: the Independent and the Cooperative structure. Previous results had stated that the Cooperative structure always outperforms the Independent one. However, with a limited number of samples available for signal detection, we need to justify how cooperative the processing should be to maintain that statement. Toward this end we propose the Partially Cooperative structure that relaxes the Identifiability Condition (IC) of a single Rx antenna K-BA-MUD. It is concluded that the proposed structure will outperform the Fully Cooperative one in any condition, given the number of samples is small and the IC is not violated. Finally, by reducing the size of the steering vector, we also reduce its computational complexity for updating the detector parameters.
\end{abstract}

Keywords: Kalman-based BA-MUD, multiple receiver antennas, identifiability condition, complexity reduction.

\section{INTRODUCTION}

Extensive efforts had been devoted in the recent decades to The BA-MUD techniques in CDMA systems. The BAMUD becomes attractive because it requires only the intended users' information such as signatures and the timing delay, while ignoring the interferers' information.

The canonical form of the BA-MUD had been suggested in [1]. By optimizing minimum output energy (MOE) of the detector output, the detector parameters can be obtained for AWGN channel. For its adaptive implementation, LMS had been employed. Accordingly, by using a faster convergence rate and a better tracking capability filter, RLSbased BA-MUD (RLS-BA-MUD) had been proposed in [2] for AWGN channel and synchronous flat-fading channel. This detector parameters are obtained directly from the covariance matrix of the received signal through a constraint optimization approach. Some performance improvement had been reported. Later on, this technique had been extended to multipath case by imposing more accurate code constraints in [3],[4]. Another technique using subspacebased had been investigated thoroughly in [5],[6].

Recently, the Kalman-based BA-MUD (K-BA-MUD) had been proposed for AWGN and synchronous flat-fading channel in [7]. Unlike the LMS- and the RLS-BA-MUD, the K-BA-MUD had been shown to have a steady-state excess output energy (EOE) approaching zero as the number of sample increases in a stationary environment; if the number of sample is infinite, K-BA-MUD will have a similar performance to the non-blind MMSE receiver presented in [8]. Most recently, K-BA-MUD had been expanded to multipath case in [9].

Nevertheless, when the identifiability condition (IC), i.e. the condition that guarantees the technique will perform, of K-BA-MUD is violated, then the system performance will degrade. As a solution, we further develop the K-BA-MUD by employing multiple receiver $(\mathrm{Rx})$ antennas to relax that IC condition. Even though the idea of exploiting the spatialtemporal diversity had been investigated for subspace-based BA-MUD (SS-BA-MUD) in [10], it differs in which we consider only a small number of data samples available.

This paper is organized as follows. The system model used throughout this paper is similar to [6] and is given in section 2. In section 3, we investigate two K-BA-MUD Rxstructures with multiple Rx-antennas: the Independent and the Cooperative structure. An extension to the Cooperative structure, a Partially Cooperative structure is proposed and investigated. The Kalman filtering is presented in section 4, the performance analysis by means of computer simulation is given in section 5 and finally, this paper will be concluded in section 6 .

\section{SYSTEM MODEL}

Consider a $K$-user asynchronous DS-CDMA system where each user $k=\{1 \ldots K\}$ is employing a single transmitter (Tx) antenna and base station (BS) is using $Q$ Rx-antennas. 
A frame of $M$ symbols is sent by user $k$ and is given by

$$
x_{k}(t)=A_{k} \sum_{i=0}^{M-1} b_{k}[i] s_{k}\left(t-i . T_{c}-d_{k}\right)
$$

where $A_{k},\left\{b_{k}[i]\right\}$, and $d_{k}$ denote the amplitude, an independent, equiprobable binary symbol stream with interval $T_{s}$, and the transmission delay of user $k$, respectively. The normalized, complex-valued signature $s_{k}(t)$ assigned to user $k$ is supported within the interval $\left[0,(N-1) T_{c}\right]$, with $N=$ $T_{s} / T_{c}$ is the processing gain. Let the channel impulse response (CIR) from user $k$ to Rx-antenna $q$ be

$$
g_{k}^{[q]}(t)=\sum_{l=1}^{L} \alpha_{k l}^{[q]} \delta\left(t-\tau_{k l}^{[q]}\right)
$$

where $L, \alpha_{k l}^{[q]}$ and $\tau_{k l}^{[q]}$ represent the number of multipath, the complex-valued path gain and the path delay of channel from user $k$ to Rx-antenna $q$ in path $l$, respectively. By using (1) and (2), the received signal component at the Rx-antenna $q$ due to user $k$ can be written as

$$
r_{k}^{[q]}(t)=\sum_{i=0}^{M-1} b_{k}[i] \underbrace{A_{k} s_{k}\left(t-i . T_{c}-d_{k}\right) \star g_{k}^{[q]}(t)}_{h_{k}^{[q]}\left(t-i T_{s}\right)}
$$

where $\star$ is a convolution process and $h_{k}^{[q]}(t)$ denotes the effective signature for the corresponding channel.

For the sake of simplicity, it is assumed that $\tau_{k l}^{[q]}=$ $\tau_{k l}, \forall q \in\{1, Q\}$ and that $\tau_{k l}$ and $d_{k}$ are chip-synchronous. And since the received signal is in-line with chip-interval, the received signal needs to be sampled only at a chip-rate. Allow $\iota_{k}=\left\lceil\frac{d_{k}+\tau_{k L}+T_{c}}{T_{s}}\right\rceil$ be the maximum delay spread of user $k$, then the $n$-th received signal sample at Rx-antenna $q$ during $i$-th symbol due to all users can be written as

$$
r^{[q]}[i, n]=\sum_{k=1}^{K} \sum_{j=0}^{\iota_{k}} h_{k}[i, n]^{[q]} b_{k}[i-j]+\eta^{q}[i, n]
$$

where $\eta^{q}$ is a complex-valued zero-mean gaussian noise at $\mathrm{Rx}$-antenna $q$ and is assumed to be locally un-correlated and also be independent of the received signal. Let $\iota=$ $\max _{k \in\{1, K\}} \iota_{k}$ and $b_{1}[i]$ be the maximum delay spread of all users and the desired bit, respectively. The received signal vector during symbol period at Rx-antenna $q$ is given by ${ }_{r}^{[q]}[i]=\left[r^{[q]}[i, 0] \ldots r^{[q]}[i, N-1]\right]^{T}$. Then, by stacking $J$ successive symbols, we define

$$
\begin{aligned}
\underline{\mathbf{r}}^{[q]}[i]= & {\left[\underline{r}^{[q] T}[i] \ldots \underline{r}^{[q] T}[i+J-1]\right]^{T} } \\
= & \tilde{\mathbf{h}}_{1}^{[q]} b_{1}[i]+\tilde{\mathbf{H}}_{1}^{[q]} \underline{\tilde{\mathbf{b}}}_{1}[i]+ \\
& \sum_{k=2}^{K} \breve{\mathbf{H}}_{k}^{[q]} \mathbf{B}_{k}[i]+\underline{\boldsymbol{\eta}}^{[q]}[i]
\end{aligned}
$$

where $\underline{\tilde{\mathbf{h}}}_{1}^{[q]}=\left[\underline{h}_{1}^{[q] T}[0] \ldots \underline{h}_{1}^{[q] T}[\iota] \quad \underline{0}^{T}\right]^{T}$ denote the stacked effective signature for $b_{1}[i]$ at Rx-antenna $q$. The second part and the third part of (5) denote the corresponding ISI and MAI, respectively, while the fourth part is the stacked gaussian noise at $\mathrm{Rx}$-antenna $q$.

\section{K-BA-MUD WITH MULTIPLE RX ANTENNAS}

In this section, we present two receiver structures to form the K-BA-MUD with multiple Rx-antennas. Since user 1 is the intended user, a canonical form for the K-BA-MUD detector, suggested in [7], can be written as

$$
\underline{\mathbf{f}}_{1}[i]=\underline{\tilde{\mathbf{h}}}_{1}-\mathbf{H}_{1, n u l l} \underline{w}_{1}[i]
$$

where $\underline{\mathbf{f}}_{1}[i], \underline{w}_{1}[i]$, and $\mathbf{H}_{1, \text { null }}$ indicate the detector vector, the steering vector, and the matrix that spans the null-space of $\underline{\tilde{\mathbf{h}}}_{1}$, respectively. In BS, the use of multiple Rx-antennas is expected and the processing power is allowed to be much larger than that of the mobile terminal.

\subsection{The Independent Structure (I-K-BA-MUD)}

With this structure, each Rx-antenna $q$ will have its own K-BA-MUD detector defined in (6), $\underline{\mathbf{f}}_{1}^{[q]}[i]$. This arrangement will only exploit the spatial diversity and the Maximum Likelihood (ML) decision rule for this structure is given by

$$
\hat{b}_{1}[i]=\operatorname{sign}\left\{\sum_{q=1}^{Q} \underline{\mathbf{f}}_{1}^{[q] H}[i] \underline{\mathbf{r}}^{[q]}[i]\right\}
$$

We can easily see that the K-BA-MUD from each Rx-antenna $q$ works exclusively to other Rx-antenna $\widetilde{q} \in\{1, \ldots, Q\} \backslash$ $q$, therefore its performance depends solely on the ability of the kalman filtering procedure to detect the signal. Increasing the number of Rx-antennas will enhance the signal power, which in turn will enhance the system performance. However, shall the IC of the K-BA-MUD from all $\mathrm{Rx}$-antennas is violated, increasing the number of Rx antennas will only raise the noise power, thus causing the detrimental effect on system performance. Notice that this structure does not relax the IC of the K-BA-MUD.

\subsection{The Cooperative Structure}

The Cooperative structure will exploit the spatial-temporal diversity by combining all information gathered from the Rx-antenna $q=\{1, \ldots, Q\}$. By doing so, the IC of the K-BA-MUD will be relaxed. 


\subsubsection{The Fully Cooperative Structure (FC-K-BA-MUD)}

This structure incorporates the obtained information from all Rx-antenna $q=\{1, \ldots, Q\}$ into a single K-BA-MUD detector to perform signal detection. To apply this structure, we define the following vectors:

$$
\underline{\tilde{\mathbf{h}}}_{1}[i]=\left[\begin{array}{c}
\tilde{\mathbf{h}}_{1}^{[1]} \\
\vdots \\
\tilde{\mathbf{h}}_{1}^{[Q]}
\end{array}\right] ; \quad \underline{\mathbf{r}}[i]=\left[\begin{array}{c}
\underline{\mathbf{r}}^{[1]}[i] \\
\vdots \\
\underline{\mathbf{r}}^{[Q]}[i]
\end{array}\right]
$$

and then use them in (6) to form the K-BA-MUD detector. It is easy to see that both the combined received signal vector $\underline{\mathbf{r}}[i]$ and the combined effective signature vector $\underline{\tilde{\mathbf{h}}}_{1}[i]$ will have length of $J N Q$, and by using (10), the steering vector will have size of $J N Q$ too. The ML decision rule for this arrangement is given by

$$
\hat{b}_{1}[i]=\operatorname{sign}\left\{\underline{\mathbf{f}}_{1}^{H}[i] \underline{\mathbf{r}}[i]\right\}
$$

With this structure, we can anticipate that the more Rxantennas is employed, the bigger the steering vector size will be and the longer time it needs to converge.

It is a general knowledge that the cooperative structure always outperforms the independent structure as long as the number of data samples $M$ tends to infinity. However, in a high data-rate wireless application where the channel may vary rapidly, thus only a small $M$ is available. Therefore it is a challenging task for BA-MUD to achieve a good performance even with a small $M$; the performance of BA-MUD is now a function of $M$.

\subsubsection{The Partially Cooperative Structure (PC-K-BA-MUD)}

With a considerably small $M$, a more efficient configuration needs to be found, especially when the IC for a single $\mathrm{Rx}$-antenna K-BA-MUD is violated. Therefore, instead of utilizing all Rx-antennas available in a single K-BA-MUD, we suggest to partially integrate the information gathered at some Rx-antennas into clusters, hence it is named the Partially Cooperative structure. Each cluster will process the received signal independently from other clusters, while all Rx-antenna in its corresponding cluster will process their received signal cooperatively.

Let $L_{c l}$ and $N_{c l}=\left(Q / L_{c l}\right)$ be the cluster's size and the number of clusters in the system. Denote

$$
\begin{aligned}
\underline{\tilde{\mathbf{h}}}_{1, \vartheta}[i] & =\left[\begin{array}{c}
\underline{\tilde{\mathbf{h}}}_{1}^{\left[(\vartheta-1) \times L_{c l}+1\right]} \\
\vdots \\
\tilde{\underline{\mathbf{h}}}_{1}^{\left[\vartheta \times L_{c l}\right]}
\end{array}\right] ; \\
\underline{\mathbf{r}}_{\vartheta}[i] & =\left[\begin{array}{c}
\underline{\mathbf{r}}^{\left[\left[(\vartheta-1) \times L_{c l}+1\right]\right]}[i] \\
\vdots \\
\underline{\mathbf{r}}^{\left[\vartheta \times L_{c l}\right]}[i]
\end{array}\right]
\end{aligned}
$$

where $\vartheta \in\left\{1, L_{c l}\right\}$ denotes the cluster index. The ML decision rule for $b_{1}[i]$ for this structure is given by

$$
\hat{b}_{1}=\operatorname{sign}\left\{\sum_{c l=1}^{2} \underline{\mathbf{f}}_{1, c l}^{[q] H}[i] \underline{\mathbf{r}}_{c l}^{[q]}[i]\right\}
$$

It is easy to inspect that by clustering only some $\mathrm{Rx}$-antennas together, the steering vector will be smaller than the FCK-BA-MUD's and thus, the less time needed to converge, provided the IC, relaxed by integrating $N_{c l} \mathrm{Rx}$-antennas together in a cluster, is still not violated.

\section{KALMAN FILTERING ALGORITHM}

Kalman filter has the ability to span the received signal from the infinite past up to the present time. That characteristic makes it the optimum filter in MMSE sense. In this section, we will construct the parameters needed to form (6) and the procedure for updating it.

From (6), we can see that the adaptive part of the KBA-MUD detector is the steering vector $\underline{w}_{1}[n]$, whose size depends on the matrix $\mathbf{H}_{1, \text { null }}$. The only requirement for obtaining $\mathbf{H}_{1, \text { null }}$ is that $\underline{\mathbf{h}}_{1}^{H} \mathbf{H}_{1, \text { null }}=\underline{0}$. As suggested in [9], we can define

$$
\mathbf{H}_{1, n u l l}=\mathbf{I}-\underline{\tilde{\mathbf{h}}}_{1}\left(\underline{\tilde{\mathbf{h}}}_{1}^{H} \underline{\tilde{\mathbf{h}}}_{1}\right)^{-1} \underline{\tilde{\mathbf{h}}}_{1}^{H}
$$

with this method, $\mathbf{H}_{1, \text { null }} \in \mathbb{C}^{(J N \times J N)}$. We re-define $\underline{\tilde{\mathbf{h}}}_{1}=$ $\left[\begin{array}{ll}\underline{h}_{1}^{T} & \underline{0}^{T}\end{array}\right]^{T}$. Let $L_{s}$ be the length of $\underline{h}_{1}$, then the null vector will have size of $\left(J N-L_{s}\right)$. Therefore by using $\underline{h}_{1} \in \mathcal{C}^{L_{s}}$, $\mathbf{H}_{1, \text { null }} \in \mathcal{C}^{L_{s} \times L_{s}}$ can be formed and the same adjustment needs to be done for (6) such that all parameters have the same length. This adjustment makes no loss in information, because we just remove the zeros out of the computation. Moreover, by reducing the size of steering vector as suggested above, we may speed up its convergence rate.

The detector output of the K-BA-MUD is given by

$$
\underbrace{\underline{\mathbf{f}}_{1}^{H}[i] \underline{\mathbf{r}}[i]}_{e[i]}=\underbrace{\tilde{\mathbf{h}}_{1}^{H} \underline{\mathbf{r}}[i]}_{\tilde{r}[i]}-\underbrace{\mathbf{r}^{H}[i] \mathbf{H}_{1, n u l l}}_{\underline{d}^{H}[i]} \underline{w}_{1}[i]
$$

Under our assumption that the CIR stays unchanged during one frame transmission, the optimum detector defined in (6) will also stay constant, and so will the steering vector $\underline{w}_{1, \text { opt }}[i]$. Therefore, we can define the state-transition (process) and measurement equation for kalman filter as follows:

$$
\begin{aligned}
\underline{w}_{1, \text { opt }}[i+1] & =\underline{w}_{1, \text { opt }}[i] \\
\tilde{r}[i] & =\underline{d}^{H}[i] \underline{w}_{1}[i]+e[i]
\end{aligned}
$$


Table 1. Computational Complexity Comparison

\begin{tabular}{|l||c|}
\hline BA-MUD Techniques & Complexity for Updating \\
\hline \hline RLS & $\mathrm{O}\left(N^{2} J^{2}\right)$ \\
SUBSPACE (MMSE,PASTd) & $\mathrm{O}\left(K N J^{2}\right)$ \\
KALMAN [9] & $\mathrm{O}\left(N^{2} J^{2}\right)$ \\
KALMAN (proposed) & $\mathrm{O}\left(L_{s}^{2}\right)$ \\
I-K-BA-MUD & $\mathrm{O}\left(Q L_{s}^{2}\right)$ \\
FC-K-BA-MUD & $\mathrm{O}\left(Q^{2} L_{s}^{2}\right)$ \\
PC-K-BA-MUD & $\mathrm{O}\left(N_{c l} L_{c l}^{2} L_{s}^{2}\right)$ \\
\hline
\end{tabular}

By using Riccati equation solver defined in [11], we can update the K-BA-MUD parameters as follows.

$$
\begin{aligned}
\underline{G}(i)= & \mathbf{K}(i, i-1) \underline{d}[i] \\
& {\left[\underline{d}^{H}[i] \mathbf{K}(i, i-1) \underline{d}[i]+\gamma\right]^{-1} } \\
\alpha(i)= & \tilde{r}-\underline{d}^{H}[i] \underline{w}_{1, o p t}(i-1) \\
\underline{w}_{1, o p t}(i)= & \underline{w}_{1, o p t}(i-1)+\underline{G}(i) \alpha(i) \\
\mathbf{K}(i+1, i)= & \mathbf{K}(i, i-1)- \\
& \underline{G}(i) \underline{d}^{H}[i] \mathbf{K}(i, i-1)
\end{aligned}
$$

In this paper, we assume the effective signature $\underline{h}_{1}$ is perfectly known a priori the signal detection. Thus, we can use the results from [7] to estimate $\gamma$ in (13) by taking $\gamma \simeq A_{1}^{2}$. For initial condition, $\mathbf{K}(1,0)=\mathbf{I}$ and $\underline{w}_{1}=\underline{0}$ had been suggested in [11].

\section{NUMERICAL RESULTS}

In this section, we provide the numerical results by means of computer simulation. Before that, we present the comparison of the computational complexity (in terms of number of multiplication) required to update the detector parameters of several BA-MUD techniques in table 1. As we can see, the SS-BA-MUD [6] has the lowest computational complexity required to update its detector parameter, namely the signal subspace, in particular when the processing gain $N$ is large. RLS-BA-MUD [2] and K-BA-MUD [7] have similar complexity, while the proposed K-BA-MUD has a lower complexity, especially when the maximum delay spread of the intended user is much smaller than the symbol interval. Furthermore, we can see that the I-K-BA-MUD requires the smallest computational complexity, while the FCK-BA-MUD is the most complex one. Note that there is a trade-off between complexity and the strictness of the IC.

Throughout the simulation, we set $N=15$, CIR is 3-tap rayleigh fading channel, the transmission delay is uniformly distributed in the interval $0,8 T_{c}$, the number of data samples is $M=1500$ and the intended user's signal is received with $\mathrm{SNR}=10 \mathrm{~dB}$, or otherwise stated. The signal power is set to a constant $A_{1}^{2}=1$, whilst the noise power is adjusted

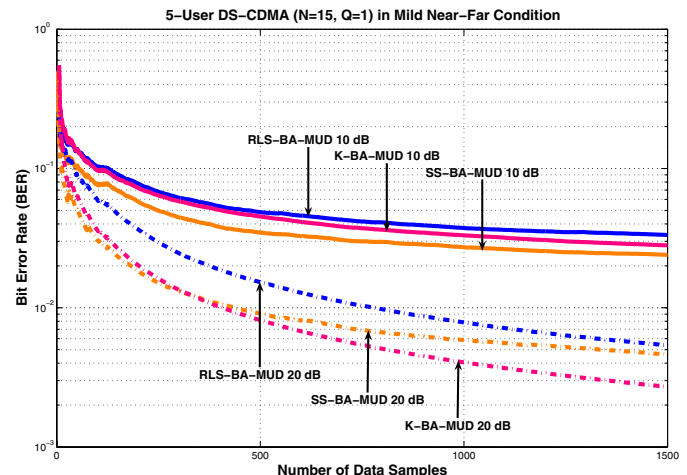

Fig. 1. BER Performance Several BA-MUD Techniques

accordingly. For example the noise power $\sigma^{2}=0.1$ when $\mathrm{SNR}=10 \mathrm{~dB}$. The results presented are the average over 200 Monte-Carlo simulation runs.

First, the simulation is run under the condition where the IC of the K-BA-MUD is held. The system scenario is a mild near-far condition, with two interferers transmitting with power $A_{2}^{2}=A_{3}^{2}=10$ and another interferers having $A_{4}^{2}=A_{5}^{2}=100$. The BER performance of three batch BAMUD techniques: the RLS-BA-MUD [4], the SS-BA-MUD [6], and the K-BA-MUD are compared. Because the RLSBA-MUD and the SS-BA-MUD depend highly on the accuracy of the covariance matrix of the received signal while the K-BA-MUD does not, therefore for fairness since the CIR is a priori known perfectly, we set the learning period (after which the error is measured)) to 100 samples to get a good approximation of that matrix. The forgetting factor used in the RLS-BA-MUD and the SS-BA-MUD is 0.997. The results are depicted in fig.1. For $S N R=10 \mathrm{~dB}$ the SSBA-MUD performs the best while for $\mathrm{SNR}=20 \mathrm{~dB}$, the KBA-MUD outperforms others. Another point to make is that the K-BA-MUD tends to perform better as $M$ increases, as noted in [7], while other techniques seem to have the socalled error-floor due to the in-accuracy of the covariance matrix of the received signal. Hence, for $M \rightarrow \infty$, we can expect the K-BA-MUD to have a similar performance to the non-blind MMSE receiver.

Next, the use of multiple Rx antennas in conjunction with the K-BA-MUD is simulated in the moderate nearfar condition (the IC is still held): $A_{2}^{2}=10, A_{3}^{2}=100$, $A_{4}^{2}=A_{5}^{2}=1000$. The learning period is set only to 2 symbols to see actual convergence rate of the K-BA-MUD. We can see from fig. 2, for BS with $2 \mathrm{Rx}$-antennas, the I-K-BA-MUD performs better compared to the FC-K-BAMUD when $M \leq 700$, while after that the FC-K-BA-MUD does. For BS employing 4 Rx-antennas, with the FC-K-BAMUD is expected to need much more time to perform the detection, hence, is definitely inferior to other structures in 


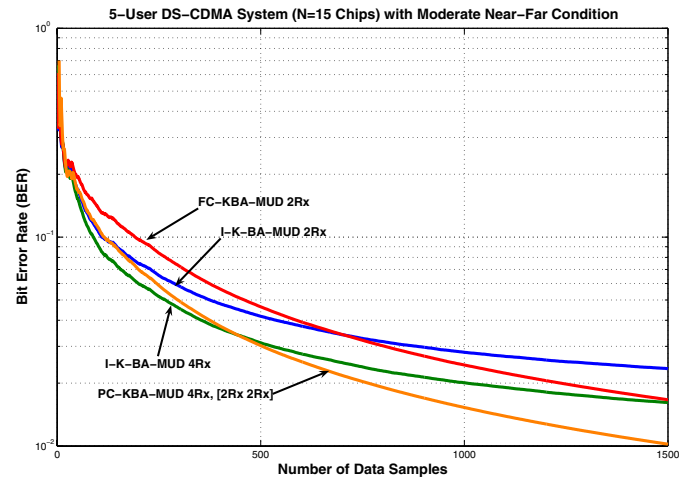

Fig. 2. BER Performance K-BA-MUD with Multiple RxAntennas in a Moderate Near-Far Condition

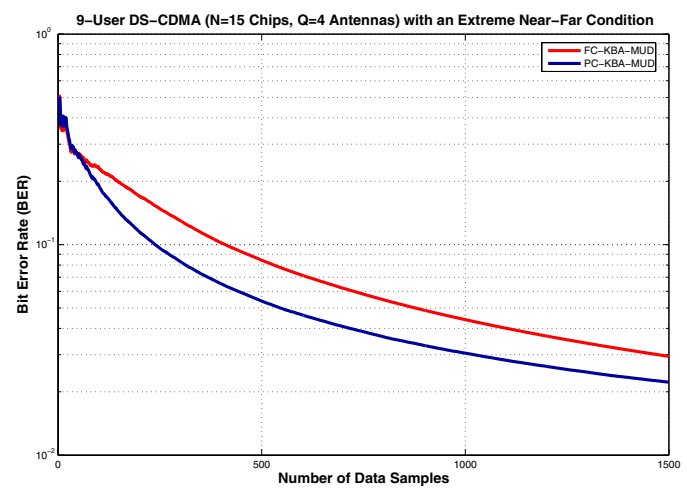

Fig. 3. BER Performance K-BA-MUD with Multiple RxAntennas in an Extreme Near-Far Condition

small $M$, hence the proposed PC-K-BA-MUD is the dominant compared to the I-K-BA-MUD after $M=450$.

Following, the system is set to have such an extreme near-far effect condition: $A_{2}^{2}=A_{3}^{2}=A_{4}^{2}=10, A_{5}^{2}=$ $A_{6}^{2}=100, A_{7}^{2}=A_{8}^{2}=1000$, and $A_{9}^{2}=10000$, that the IC is violated and the I-K-BA-MUD fails completely. From fig. 3 , it is shown that the PC-K-BA-MUD is superior to the FCK-BA-MUD for rather small $M$. Though, we should expect that the PC-K-BA-MUD is always the best as $M \rightarrow \infty$.

\section{CONCLUSION}

In this paper, the K-BA-MUD is compared with the RLSBA-MUD and the SS-BA-MUD in terms of BER performance with limited number of data samples $M \leq 1500$. As $M$ increases, the K-BA-MUD will outperform the other BA-MUD techniques. Moreover, there are certain conditions where the IC of K-BA-MUD is violated. Therefore the IC is relaxed by employing multiple $\mathrm{Rx}$ antennas in conjunction with the K-BA-MUD. The I-K-BA-MUD will give a better performance than others, provided the IC holds for a single Rx-antenna and $M$ is very small, for example $M \leq 400$. The proposed PC-K-BA-MUD structure will outperform the FC-K-BA-MUD in any condition, provided limited $M$ is considered and the IC is held in any clusters. Furthermore, the PC-K-BA-MUD has complexity considerably lower than the FC-K-BA-MUD.

\section{REFERENCES}

[1] M. Honig, U. Madhow, and S. Verdú, "Blind adaptive multiuser detection," IEEE Trans. Inform. Theory, vol. 41, no. 4, Jul. 1995.

[2] H.V. Poor and X. Wang, "Code-aided interference suppresion for DS/CDMA communications-part II: Parallel blind adaptive implementations," IEEE Trans. Commun., vol. 45, no. 9, Sep. 1997.

[3] M.K. Tsatsanis and Z. Xu, "Performance analysis of minimum variance CDMA receivers," IEEE Trans. Signal Processing, vol. 46, no. 11, Nov. 1998.

[4] Z. Xu, P. Liu, and X. Wang, "Blind multiuser detection: from MOE to subspace methods," IEEE Trans. Signal Processing, vol. 52, no. 2, Feb. 2004.

[5] X. Wang and H.V. Poor, "Blind multiuser detection: A subspace approach," IEEE Trans. Inform. Theory, vol. 44, no. 2, Mar. 1998.

[6] X. Wang and A.Høst-Madsen, "Group-blind multiuser detection for uplink CDMA," IEEE J. Select. Areas Commun., vol. 17, no. 11, Nov. 1999.

[7] X.D. Zhang and W. Wei, "Blind adaptive multiuser detection based on kalman filtering," IEEE Trans. Signal Processing, vol. 50, no. 1, Jan. 2002.

[8] S. Verdú, Multiuser Detection, New York: Cambridge Univ. Press, 1998.

[9] X. Liu, K.C. Teh, and E. Gunawan, "Blind adaptive kalman filter-based multiuser detector over a multipath fading channel," IEEE Commun. Let., vol. 8, no. 6, Jun. 2004.

[10] D. Reynolds, X. Wang, and H.V. Poor, "Blind adaptive space-time multiuser detection with multiple transmitter and receiver antennas," IEEE Trans. Signal Processing, vol. 50, no. 6, Jun. 2002.

[11] S. Haykin, Adaptive Filter Theory, New Jersey: Prentice-Hall, Inc., 3rd edition, 1996. 